\title{
Principal Scientific Results of the Surveyor 3 Mission
}

\author{
L. D. Jaffe, ${ }^{1}$ S. A. Batterson, ${ }^{2}$ W. E. Brown, JR., ${ }^{1}$ E. M. Christensen, ${ }^{1}$ \\ D. E. Gault, ${ }^{3}$ J. W. Lucas, ${ }^{1}$ R. H. Norton, ${ }^{1}$ R. F. Scott, ${ }^{4}$ E. M. \\ Shoemaker, ${ }^{5}$ G. H. Sutton, ${ }^{6}$ and A. L. Turkevich ${ }^{7}$
}

\begin{abstract}
The fine lunar surface material at the Surveyor 3 landing site has about $3 \times 10^{3}$-dyne $/ \mathrm{cm}^{2}$ cohesion, $35^{\circ}$ angle of internal friction, $3 \times 10^{5}$-dyne $/ \mathrm{cm}^{2}$ static bearing capacity. A small rock withstood a local pressure of $2 \times 10^{7}$ dynes $/ \mathrm{cm}^{2}$. Soil strength and density increase significantly at depths of a few centimeters. Exposed surface has a considerably higher albedo than the material just below it. The photometric function changed when the surface was slightly compressed. Fine surface material appears to be gradually moving downslope.
\end{abstract}

Data returned by Surveyor 3 during and after landing on the lunar surface provided significant additional information about the moon. Of particular interest are the results on mechanical properties of the lunar surface and the comparisons of terrain and microfeatures inside and outside a crater, made possible by observations from Surveyors 1 and 3.

This paper outlines the major scientific findings derived from Surveyor 3 data and is based on information obtained by varied methods, which are described in more detail in the following papers.

\section{Mechanical Propertigs of Lunar Surface Material}

During the Surveyor 3 landing events the footpads formed craters and made imprints on the lunar surface. Associated with the footpad craters were patterns of ejected material, indicative of the direction of spacecraft motion. The craters, imprints, and ejecta have been used in analyses of lunar soil properties. Sev-

\footnotetext{
${ }^{1}$ Jet Propulsion Laboratory, California Institute of Technology, Pasadena, California 91103.

${ }^{2}$ Dynamic Loads Division, NASA Langley, Research Center, Hampton, Virginia 23361.

${ }^{8}$ Space Science Division, NASA Ames Research Center, Moffett Field, California 94035.

California Institute of Technology, Pasadena, California 91103.

'Department of Astrogeology, U. S. Geological Survey, Flagstaff, Arizona 86001.

- Hawaii Institute of Geophysics, University of Hawaii, Honolulu, Hawaii 96822.

'Enrico Fermi Institute, University of Chicago, Chicago, Illinois 60637.
}

eral other methods were also used to derive the mechanical properties of the lunar soil: analyses and simulations of the landing dynamics, analyses of the soil mechanics surface sampler (SMSS) experiment, and slopes of small natural craters. All methods considered, the following ranges of soil properties seem to be compatible with the observations and to represent the differences in techniques of analysis and, possibly, variation in the lunar soil propereties from place to place:

1. Cohesion range, $1.5 \times 10^{3}$ to $7 \times 10^{3}$ dynes/cm ${ }^{2}$.

2. Angle of internal friction range, $35^{\circ}$ to $40^{\circ}$.

3. Static bearing capacity range, $2 \times 10^{6}$ to $5 \times 10^{5}$ dynes $/ \mathrm{cm}^{2}$.

Note that static bearing capacity increases with bearing size.

The SMSS picked up a clod, or aggregate of lunar soil grains, that was crushed when the scoop door was closed and a rock fragment that was not crushed. It is estimated that a pressure of approximately $2 \times 10^{7}$ dynes $/ \mathrm{cm}^{2}$ was applied to the rock when the scoop was closed. The rock did not break under this stress.

Lunar soil was observed to remain in the SMSS scoop with the door fully open until it was jarred free by the SMSS motions or impact. It is possible that the soil adhered to the scoop [Scott and Roberson, 1968], or it may be that moldable, slightly cohesive soil adapted itself to the inside shape of the scoop and was jammed in place by a trenching operation, without adhesion [Gault et al., 1968]. 
Vibrations of the spacecraft after the third touchdown have been analyzed to give an indication of the elastic shear or rigidity modulus of the surface soil [Christensen et al., 1968].

\section{Macroscopic Features of Local Terrain}

Surveyor 3 landed in a subdued crater that had a low rounded rim. The crater is slightly more than 200 meters in diameter and about 15 meters deep. The spacecraft is situated on the inner east wall of the crater, about halfway between the center of the crater and the rim crest. It is inclined $14.7^{\circ} \pm 0.1^{\circ}$ toward the west. The selenographic coordinates of the landed spacecraft are $2.94^{\circ} \mathrm{S}$ latitude, $23.34^{\circ} \mathrm{W}$ longitude relative to selenodetic control adopted by the Aeronautical Chart and Information Center.

The small craters observed in the Surveyor 3 pictures range in diameter from about $10 \mathrm{~cm}$ to more than 20 meters. Most craters smaller than 3 meters in diameter are relatively shallow and are nearly rimless or have very subdued rims. Approximately $25 \%$ of the craters that range from 3 to about 13 meters in diameter have distinctly raised rims and relatively steep walls. The other craters in this size range have subdued forms. The rims of most craters are composed of materials similar in texture to parts of the surface between the craters. Most craters have a normal cup shape, with walls and floors concave upward. One prominent crater more than 20 meters across, located near the center of the main crater, is dimpleshaped, however. Most of the craters appear to be irregularly or nearly randomly distributed over the rim, walls, and bottom of the main crater, but some of the very shallow rimless craters are aligned approximately in the northsouth direction. The size/frequency distribution of the craters at the Surveyor 3 landing site is very similar to the mean distribution observed on the lunar plains by Rangers 7 through 9.

Bright pieces of fragmental debris are scattered irregularly over most of the visible parts of the lunar surface. Most of the observed fragments are relatively angular, but some well-rounded fragments, which generally appear to be deeply buried in the lunar surface, are also present. Generally, the fragments are equant in shape, but some are distinctly tab- ular and a few have the form of sharp narrow wedges. Two prominent strewn fields of blocky debris were observed around two craters, 13 and 15 meters across, at the Surveyor 3 site. One of these craters has a sharp raised rim and the other a more subdued rounded rim.

Fragments close to the spacecraft have a peculiar grainy, knobby, or pitted texture. Some fragments, notably pieces associated with a strewn field of blocks to the northeast of the spacecraft, have smooth, nearly planar, faces, as though they were broken along pre-existing joints or fractures. Some large blocks, most of which are tabular in form, appear to be laminated or to contain planes of weakness parallel to the long dimensions of the blocks.

Most of the fragments at the surface of the Surveyor 3 landing site are evidently part of a layer of particulate material of low cohesion. The layer is at least 1 meter thick along the upper parts of the wall of the main crater in which Surveyor 3 landed and may be much thicker near the center of the crater. The presence of coarse blocky ejecta from superposed craters on the northeast rim and high on the southwest wall indicates that coarser fragments or more coherent material is present at depths of a few meters on or near the rim of the main crater. No strewn fields of blocks or blocky crater rims are found near the bottom of the main crater. This may indicate that the base of the relatively fine-grained debris layer is deeper than any of the craters present there.

Lunar surface brightness temperatures sensed by Surveyor 3 during the lunar day correspond to a thermal parameter $\gamma$ greater than 400 in cgs units; this value is in agreement with earthbased data. Also during the day, lunar surface directional thermal emission was sensed and later found to be consistent with earth-based data. Temperature measurements taken during eclipse permit a more accurate determination of the thermal parameter and indicate a value of approximately 400; this value is not in agreement with the value of 1420 obtained by earth-based eclipse measurements for the landing site region. The Surveyor 3 temperature data lag the earth-based measurements during the eclipse.

The average radar cross section of the moon, as measured from the earth, closely approxi- 
mates the values measured by Surveyor radars over small areas (250-meter radius).

\section{Characteristics of Local Microstructure}

From the strain gage data, it is concluded [Christensen et al., 1968] that the undisturbed lunar surface density to a depth of $0.5 \mathrm{~cm}$ is not greater than about $1.2 \mathrm{~g} / \mathrm{cm}^{3}$ and that the material to this depth compressed during the impact to a density of 1.7 to $1.8 \mathrm{~g} / \mathrm{cm}^{3}$. Analysis of both SMSS and the touchdown measurements, from a soil mechanics viewpoint, led to a density estimate of about $1.5 \mathrm{~g} / \mathrm{cm}^{\mathrm{s}}$ for the top few centimeters of lunar soil [Scott and Roberson, 1968]. In penetrations to this depth it was observed that the material appeared to be relatively incompressible. It would appear that the two computed ranges of density can be reconciled if a lunar soil profile of increasing density is postulated. The increase must be fairly rapid to explain the observations, with a density of $1 \mathrm{~g} / \mathrm{cm}^{3}$ or less in the top few millimeters, increasing to about $\mathbf{1 . 5}$ $\mathrm{g} / \mathrm{cm}^{8}$ by a depth of a few centimeters and to an asymptotic value of perhaps 1.8 to 2.0 $\mathrm{g} / \mathrm{cm}^{\mathbf{3}}$ at a depth of perhaps 5 to $10 \mathrm{~cm}$, based on limiting lower-porosity considerations and an assumed solid component density of 3 $\mathrm{g} / \mathrm{cm}^{\mathrm{s}}$. The corresponding porosity range is from about 0.7 at the surface to 0.35 at depths greater than $10 \mathrm{~cm}$.

The SMSS experiments indicated an increase in the strength of the lunar soil with depth, an effect that became apparent at depths of 4 or $5 \mathrm{~cm}$. At 5- to 10-cm depths, considerable difficulty was encountered in deepening the trenches with the SMSS, although the deeper material was visually identical to the soil at shallower depths. The soil mechanical properties are not independent of one another or of density. Thus, it should be observed that an increase in density of the soil with depth will imply both an increase in the friction angle of the soil and an increase in the cohesion resulting from a greater number of contacts between particles. The cohesion may also differ with depth because of physicochemical reasons.

Individual particles of undisturbed surface material visible to the Surveyor 3 camera ranged in size from blocks 4 meters long down to the limit of resolution. One to ten particles per square centimeter larger than $1 \mathrm{~mm}$ in diameter were noted. The observed cumulative frequency was an exponential function of particle diameter, the exponent being about $\mathbf{- 2 . 5}$. Laboratory tests suggest that some fine particles were probably missed in counting, and the exponent for cumulative frequency per unit volume probably is higher. In the two strewn fields, around craters of from 13 to 15 meters in diameter, $10-\mathrm{cm}$ to 1 -meter blocks were much more frequent than elsewhere. Less than $15 \%$ of the surface is covered with particles larger than $1 \mathrm{~mm}$ in diameter. The surface retained a good imprint of footpad ridges about $60 \mu \mathrm{m}$ high. This observation (together with laboratory simulations, the appearance of the disturbed lunar surface, mechanical property measurements, and photometric observations of the footpad imprint and of the undisturbed surface) suggests that a substantial fraction of the surface material consists of particles smaller than $60 \mu \mathrm{m}$ in diameter and an appreciable fraction $(>10 \%)$ probably consists of particles smaller than $10 \mu \mathrm{m}$.

A preliminary search for color differences (by color reconstitution methods) revealed no determinable differences in color among various coarse blocks, the fine-grained matrix of the surface, or fine-grained material disturbed by the SMSS. All the materials observed on the Iunar surface are gray.

The estimated normal luminance factor (normal albedo) of an undisturbed part of the lunar surface next to footpad 2 of the Surveyor 3 spacecraft is $8.5 \pm 2 \%$. The smooth walls and bottom of the footpad imprints are different photometrically from the undisturbed lunar surface and from material ejected by the footpads. Observations of the imprint of footpad 2 (next to the spacecraft) indicate that this smooth surface is more like a Lambertian surface than the undisturbed lunar surface is. About 30\% more light is emitted toward the eastward-facing camera from the smooth imprint surface than from nearby undisturbed parts of the moon at phase angles of $40^{\circ}$ to $60^{\circ}$ in the lunar morning. In the lunar afternoon the imprint is not significantly brighter than the undisturbed surface. This indicates that the very small irregularities between the grains have been partly smoothed out or filled in by pressure of the smooth footpad.

Other disturbances of the lunar surface by 
Surveyor 3 exposed darker material at depths of a few centimeters or less. The fine-grained particulate debris of the lunar surface is probably 20 to $30 \%$ lower in albedo at depths of only a fraction of a millimeter beneath the optically observed undisturbed surface. Coarse particles protruding above the general level of the surface have a higher albedo than the fine-grained matrix of the surface.

\section{EFFects of Vernther ENGINe ExHadsT}

The vernier engines continued to operate throughout the first and second landings and until shortly before the touchdown of the third landing. This operation produced no noticeable effect on the spacecraft, other than on the television camera mirror. Glare was produced on part of the mirror when the sun, or large amounts of reflected sunlight, illuminated it. It is suspected that this part of the mirror was contaminated during the landing by the deposition of lunar material, by pitting, or by the deposition of exhaust gas. A lunar surface feature visible at the second landing site could possibly have been caused by vernier engine exhaust erosion. Good correspondence between the outer-face temperatures of electronic compartments of Surveyor 3 and Surveyor 1 for a given sun incidence angle indicates that there was no thermally significant layer of dust on the faces. Pictures of the top of one compartment show a few specks of material but no significant coverage. Thus, contamination of the spacecraft induced by vernier engines is not expected to be a major problem in future missions.

\section{Nonstatic Nature of Lonar Surface}

Many of the observations made during the Surveyor 3 mission can be interpreted as exhibiting lunar phenomena at different stages of their life histories. The observations provide evidence of the nonstatic nature of the lunar surface, even though the changes are occurring over a long period of time.

For example, one of the craters has a sharp raised rim and is associated with rocks of large angularity perched on top of the local landscape. It is interpreted to be young in relation to another crater nearby that has a rounded rim and less prominent debris. Similarly, examination of rocks in the vicinity of Surveyor 3 leads to a classification in terms of increasing roundness (or lack of angularity), which is interpreted as a measure of the time of exposure to abrading influences. The blocks associated with the subdued (older) crater are significantly rounder than the rocks associated with the raised-rim crater. The relation of rocks to the lunar surface in their immediate vicinity suggests a chronology in which freshly produced rocks are perched on top of the surface and older rocks are more or less buried. This correlates well with the roundness of the rocks and the nature of the neighboring craters. In addition, the fortunate landing of Surveyor 3 on a slope provided pictures showing that this burying process proceeds faster on the uphill side of a rock than on the downhill side.

The observation that disturbed lunar surface material is generally darker than undisturbed surface is evidence for a different type of process that occurs on the moon. Coarse fragments protruding above the surface, as well as the one excavated rock, have a higher albedo, however, than the fine-grained matrix of the surface. Because the surface material is continually being churned up by meteorite impacts, some process (confined in action to the surface) must be continually changing the photometric properties of the exposed particles.

\section{Relation of Properties at Surveyor 1 and SuRveror 3 Landing Sites}

The Surveyor 1 landing site was a relatively flat smooth mare surface encircled by hills and low mountains, whereas the Surveyor 3 landing site was in a subdued crater greater than 200 meters in diameter. Comparisons of the pictures obtained at these two different sites indicate that the smaller craters and the fragmental debris are similar in distribution of shape and size. The majority of the craters at both sites are inferred to be of impact origin. One difference between the two sites is in the deposition of the granular material around the more rounded rocks, which are generally believed to be buried deeper in the lunar surface than the more angular rocks are. At the Surveyor 1 site the contact line between the more rounded rocks and the surface is about in the same plane as the lunar surface, although a fillet of fine material can be ob- 
served. This indicates that processes that generally result in a smooth deposition are at work. At the Surveyor 3 site, however, the fillet of material around some of the more rounded rocks tends to be higher on the uphill end than on the downhill end, indicating a downhill movement of the fine material, as mentioned previously.

Apparent soil similarities for the top few centimeters at the Surveyor 1 and Surveyor 3 landing sites are:

1. Static bearing capability is within the range from $2 \times 10^{8}$ to $6 \times 10^{5}$ dynes $/ \mathrm{cm}^{2}$ for a footpad penetration of 2.5 to $5 \mathrm{~cm}$. This range is derived from computer simulation of shock absorber force histories and footpad penetrations during landing.

2. Material disturbed by the footpads consists of clumps or clods formed of particulate material smaller than the resolution of the camera, a fine spray thrown a meter or more, and smoothed areas that retain the compressed contact area.

3. Noncompressed disturbed material is darker than the undisturbed surface.

4. The material has little compressibility.

5. There is general homogeneity at the positions of the second and third landings of Surveyor 3 and at the landing site of Surveyor 1 .

6. Cohesion and angle of internal friction at the two sites probably are comparable.

Lunar surface brightness temperatures sensed during the day by Surveyors 1 and 3 were essentially identical. A thermal parameter value of approximately 800 from Surveyor 1 post-sunset data is significantly different from the approximately 400 from Surveyor 3 eclipse data.

The variation in radar cross section between Surveyor 1 and Surveyor 3 tracks, about $5 \mathrm{~km}$ long, is relatively small (within $\pm 20 \%$ ) except for a few anomalies related to surface features.

\section{Astronomical Observations}

Surveyor 3 pictures of the eclipse of the sun by the earth revealed a bright region in the refraction halo surrounding the earth, which was correlated with the position of the sun, and a series of bright beads over regions of the earth largely clear of clouds. Clouds usually occulted the refracted rays of the sun, most of which pass through the low atmosphere at the limb; the beads occurred in the depressions in the optical silhouette of the earth. Preliminary reduction of the color of the refracted light showed that the brightest region, near the position of the sun, exhibited a correlated color temperature close to $4800^{\circ} \mathrm{K}$. The color temperature generally was lower for light that follows paths of greater atmospheric absorption.

Preliminary analysis of Surveyor 3 pictures of the partially illuminated earth revealed colors similar to the colors recorded by the Mercury and Gemini astronauts during orbit.

\section{ReFerences}

Christensen, E. M., S. A. Batterson, H. E. Benson, R. Choate, L. D. Jaffe, R. H. Jones, H. Y. Ko, R. L. Spencer, F. B. Sperling, and G. H. Sutton, Lunar surface mechanical properties at the landing site of Surveyor 3, J. Geophys. Res., 79, this issue, 1968.

Gault, D. E., R. J. Collins, T. Gold, J. Green, G. P. Kuiper, H. Masursky, J. O'Keefe, R. Phinney, and E. M. Shoemaker, Lunar theory and processes, J. Geophys. Res., 79, this issue, 1968.

Scott, R. F., and F. I. Roberson, Soil mechanics surface sampler: Lunar surface tests, results, and analyses, J. Geophys. Res., 79, this issue, 1968.

(Received January 23, 1968.) 